\title{
BOUNDS ON THE DISJUNCTIVE TOTAL DOMINATION NUMBER OF A TREE
}

\author{
Michael A. Henning ${ }^{1}$ \\ Department of Pure and Applied Mathematics \\ University of Johannesburg \\ Auckland Park, 2006, South Africa \\ e-mail: mahenning@uj.ac.za \\ AND \\ VIROSHAN NAICKER ${ }^{2}$ \\ Department of Pure and Applied Mathematics \\ University of Johannesburg \\ Auckland Park, 2006, South Africa \\ and \\ Department of Mathematics \\ Rhodes University \\ Grahamstown, 6140 South Africa \\ e-mail: v.naicker@ru.ac.za
}

\begin{abstract}
Let $G$ be a graph with no isolated vertex. In this paper, we study a parameter that is a relaxation of arguably the most important domination parameter, namely the total domination number, $\gamma_{t}(G)$. A set $S$ of vertices in $G$ is a disjunctive total dominating set of $G$ if every vertex is adjacent to a vertex of $S$ or has at least two vertices in $S$ at distance 2 from it. The disjunctive total domination number, $\gamma_{t}^{d}(G)$, is the minimum cardinality of such a set. We observe that $\gamma_{t}^{d}(G) \leq \gamma_{t}(G)$. A leaf of $G$ is a vertex of degree 1 , while a support vertex of $G$ is a vertex adjacent to a leaf. We show that if $T$ is a tree of order $n$ with $\ell$ leaves and $s$ support vertices, then $2(n-\ell+3) / 5 \leq \gamma_{t}^{d}(T) \leq(n+s-1) / 2$ and we characterize the families of trees which attain these bounds. For every tree $T$, we show have $\gamma_{t}(T) / \gamma_{t}^{d}(T)<2$ and this bound is asymptotically tight.
\end{abstract}

Keywords: total domination, disjunctive total domination, trees.

2010 Mathematics Subject Classification: 05C69.

\footnotetext{
${ }^{1}$ Research supported in part by the South African National Research Foundation and the University of Johannesburg.

${ }^{2}$ Research supported in part by Rhodes University and the Claude Leon Foundation.
} 


\section{REFERENCES}

[1] D. Archdeacon, J. Ellis-Monaghan, D. Fischer, D. Froncek, P.C.B. Lam, S. Seager, B. Wei and R. Yuster, Some remarks on domination, J. Graph Theory 46 (2004) $207-210$. doi:10.1002/jgt.20000

[2] R.C. Brigham, J.R. Carrington and R.P. Vitray, Connected graphs with maximum total domination number, J. Combin. Math. Combin. Comput. 34 (2000) 81-96.

[3] V. Chvátal and C. McDiarmid, Small transversals in hypergraphs, Combinatorica 12 (1992) 19-26. doi:10.1007/BF01191201

[4] M. Chellali and T.W. Haynes, Total and paired-domination numbers of a tree, AKCE Int. J. Graphs Comb. 1 (2004) 69-75.

[5] M. Chellali and T.W. Haynes, A note on the total domination number of a tree, J. Combin. Math. Combin. Comput. 58 (2006) 189-193.

[6] F. Chung, Graph theory in the information age, Notices Amer. Math. Soc. 57 (2010) $726-732$.

[7] E.J. Cockayne, R.M. Dawes, and S.T. Hedetniemi, Total domination in graphs, Networks 10 (1980) 211-219. doi:10.1002/net.3230100304

[8] W. Goddard, M.A. Henning and C.A. McPillan, The disjunctive domination number of a graph, Quaest. Math. 37 (2014) 547-561. doi:10.2989/16073606.2014.894688

[9] M.A. Henning, A survey of selected recent results on total domination in graphs, Discrete Math. 309 (2009) 32-63. doi:10.1016/j.disc.2007.12.044

[10] M.A. Henning, Graphs with large total domination number, J. Graph Theory 35 (2000) 21-45. doi:10.1002/1097-0118(200009)35:1〈21::AID-JGT3〉3.0.CO;2-F

[11] M.A. Henning and V. Naicker, Disjunctive total domination in graphs, J. Comb. Optim., to appear. doi:10.1007/s10878-014-9811-4

[12] M.A. Henning and V. Naicker, Graphs with large disjunctive total domination number, Discrete Math. Theoret. Comput. Sci. 17 (2015) 255-282.

[13] M.A. Henning and A. Yeo, Total Domination in Graphs (Springer Monographs in Mathematics, 2013). doi:10.1007/978-1-4614-6525-6

[14] Zs. Tuza, Covering all cliques of a graph, Discrete Math. 86 (1990) 117-126. doi:10.1016/0012-365X(90)90354-K 